\title{
Generic zero-Hausdorff and one-packing spectral measures
}

\author{
Silas L. Carvalho* \\ Departamento de Matemática, UFMG, Belo Horizonte, MG, 30161-970 Brazil \\ and \\ César R. de Oliveira ${ }^{\dagger}$ \\ Departamento de Matemática, UFSCar, São Carlos, SP, 13560-970 Brazil
}

January 26, 2021

\begin{abstract}
For some metric spaces of self-adjoint operators, it is shown that the set of operators whose spectral measures have simultaneously zero upper-Hausdorff and one lower-packing dimensions contains a dense $G_{\delta}$ subset. Applications include sets of limit-periodic operators.
\end{abstract}

Key words and phrases. Self-adjoint operators, spectral measures, upper-Hausdorff dimension, lowerpacking dimension.

\section{Introduction}

Let $(X, d)$ be a complete metric space of self-adjoint operators acting in a separable Hilbert sapce $\mathcal{H}$, such that convergence in the metric $d$ implies strong resolvent convergence. In three previous papers [2, 3, 4, the present authors have discussed several generic sets of families of self-adjoint operators, in some instances of the space $(X, d)$, in terms of not only spectral properties, but also of dynamical ones. In such works we have gotten, through different grounds, generic sets of operators with one-dimensional packing spectral measures, but an argument for Hausdorff dimensional properties was missing; it is one of the goals of this work to fill up this gap by presenting a result in terms of what we call fractal dimensions of the spectrum: we give contributions related to the upper-Hausdorff and lower-packing dimensions of spectral measures. In $[8]$ one may find a way to the theory of singularly continuous spectra, dimensions and some of its perturbations.

Although it is already known that, for some families of self-adjoint operators, a typical (in Baire's sense) spectral measure has upper-packing dimension equal to one (see Theorem 1.1 in [4), we improve such result, in the sense that now the same result is valid for the lower-packing dimension;

\footnotetext{
*Email: silas@mat.ufmg.br

${ }^{\dagger}$ Email: oliveira@dm.ufscar.br
} 
however, as mentioned before, there was no generic result about the (upper or lower) Hausdorff dimension. The novel technical argument, encapsulated in Theorem 3.20, gives information about upper-Hausdorff dimensional properties of spectral measures; since it is immediate to adapt such ideas to obtain the counterpart lower-packing properties, we just present the details of the first case. It is also important to underline that every application of the so-called Wonderland Theorem discussed in [15], presenting dense sets of operators with pure point spectrum or absolutely continuous spectrum, can now be converted into a result about the existence of a generic set of operators whose spectral measures are zero upper-Hausdorff and one lower-packing dimensional. The upper-Hausdorff dimension of a Borel measure $\mu$ will be denoted by $\operatorname{dim}_{\mathrm{H}}^{+}(\mu)$, whereas its lower-packing dimension by $\operatorname{dim}_{\mathrm{P}}^{-}(\mu)$ (such concepts are recalled in Definition 2.15 ).

\subsection{Main results}

Next, we present our main result. It should be compared with Theorem 2.1 in [15].

Theorem 1.1 Let $0 \neq \psi \in \mathcal{H}$, let $\emptyset \neq F \subset \mathbb{R}$ be a closed set and suppose that each of the sets

- $C_{0 \mathrm{Hd}}^{\psi ; F}=\left\{T \in X \mid \operatorname{dim}_{\mathrm{H}}^{+}\left(\mu_{\psi ; F}^{T}\right)=0\right\}$,

- $C_{1 \mathrm{Pd}}^{\psi ; F}=\left\{T \in X \mid \operatorname{dim}_{\mathrm{P}}^{-}\left(\mu_{\psi ; F}^{T}\right)=1\right\}$,

is dense in $X$. Then, the set $\left\{T \in X \mid \operatorname{dim}_{\mathrm{H}}^{+}\left(\mu_{\psi ; F}^{T}\right)=0\right.$ and $\left.\operatorname{dim}_{\mathrm{P}}^{-}\left(\mu_{\psi ; F}^{T}\right)=1\right\}$ is generic in $X$.

As an illustration, we consider an application to a class of bounded discrete Schrödinger operators acting on $l^{2}(\mathbb{Z})$. For a fixed $r>0$, let $X^{r}$ be the set of operators $T$ with action

$$
(T \psi)_{n}=\psi_{n+1}+\psi_{n-1}+V_{n} \psi_{n}
$$

where the potential $v=\left(V_{n}\right)$ is an arbitrary real bilateral sequence with $\left|V_{n}\right| \leq r$ for every $n \in \mathbb{Z}$. Let $\sigma(T)$ and $\mu_{\psi}^{T}$ denote the spectrum of $T$ and its spectral measure (associated with the vector $0 \neq$ $\psi \in l^{2}(\mathbb{Z})$ ), respectively. By combining Theorem 1.1 with a specific construction presented in the proof of Theorem 4.1 in [15], we obtain the following result.

Theorem 1.2 Fix $r>0$. The set $\left\{T \in X^{r} \mid \sigma(T)=[-2-r, 2+r], \operatorname{dim}_{\mathrm{H}}^{+}\left(\mu_{\psi}^{T}\right)=0, \operatorname{dim}_{\mathrm{P}}^{-}\left(\mu_{\psi}^{T}\right)=1\right.$, for all $0 \neq \psi \in \mathcal{H}\}$ is generic in $X^{r}$.

Remark 1.3 A well-known fact about discrete Schrödinger operators in $l^{2}(\mathbb{Z})$, with action (1.1), is the existence of a common set of cyclic vectors $\left\{\delta_{0}, \delta_{1}\right\}$. Now, if for $\zeta \in\left\{\delta_{0}, \delta_{1}\right\}$ the spectral measure $\mu_{\zeta}^{T}$ is zero upper-Hausdorff dimensional, then $\mu_{\psi}^{T}$ is zero upper-Hausdorff dimensional for every vector $\psi \neq 0$ (namely, since $\mu_{\zeta}^{T}$ is supported on a set of zero Hausdorff dimension and since, for every $\psi \neq 0, \mu_{\psi}^{T}$ is absolutely continuous with respect to $\mu_{\zeta}^{T}$, then $\mu_{\psi}^{T}$ is also supported on a set of zero Hausdorff dimension), which implies that $\{T \in X \mid \sigma(T)$ is purely zero upper-Hausdorff dimensional $\}$ is a $G_{\delta}$ set (the same conclusion is valid for $\{T \in X \mid \sigma(T)$ is purely one lower-packing dimensional\}). Thus, the results stated in Theorem 1.2 are obtained after showing that the set $\left\{T \in X^{r} \mid \sigma(T)=[-2-r, 2+r], \operatorname{dim}_{\mathrm{H}}^{+}\left(\mu_{\psi}^{T}\right)=0\right.$ and $\operatorname{dim}_{\mathrm{P}}^{-}\left(\mu_{\psi}^{T}\right)=1$, for each fixed $\left.0 \neq \psi \in \mathcal{H}\right\}$, is 
generic in $X^{r}$. This is actually what one gets combining Theorem 1.1 with the aforementioned result in [15.

We also apply our results to a class of limit-periodic operators; these are discrete one-dimensional ergodic Schrödinger operators, denoted by $H_{g, \tau}^{\kappa}$, acting in $l^{2}(\mathbb{Z})$, whose action is given by (1.1), with

$$
V_{n}(\kappa)=g\left(\tau^{n}(\kappa)\right)
$$

here, $\kappa$ belongs to a Cantor group $\Omega, \tau: \Omega \rightarrow \Omega$ is a minimal translation on $\Omega$ and $g: \Omega \rightarrow \mathbb{R}$ is a continuous sampling function, i.e., $g \in \mathrm{C}(\Omega, \mathbb{R})$, the latter endowed with the norm of uniform convergence. For more details, see [1].

For each $\kappa \in \Omega$, let $X_{\kappa}$ be the set of limit-periodic operators $H_{g, \tau}^{\kappa}$ given by (1.1) and (1.2), endowed with the metric

$$
d\left(H_{g, \tau}^{\kappa}, H_{g^{\prime}, \tau}^{\kappa}\right)=\left\|g-g^{\prime}\right\|_{\infty} .
$$

We shall prove the following result.

Theorem 1.4 For each $\kappa \in \Omega$, the set $\left\{T \in X_{\kappa} \mid \sigma(T)\right.$ is purely zero upper-Hausdorff and one lower-packing dimensional\} is generic in $X_{\kappa}$.

\subsection{Countable families of pairwise commuting self-adjoint operators}

We remark that is possible to extend the result stated in Theorem 1.1 for countable families of pairwise commuting self-adjoint operators $T=\left(T_{1}, \ldots, T_{N}\right)$ acting in a separable Hilbert space $\mathcal{H}$. The joint resolution of identity is given by $E(\cdot):=\prod_{j=1}^{N} E_{j}(\cdot)$ over the rectangles of the Borel sets $\mathcal{B}\left(\mathbb{R}^{N}\right)$; here, $N$ stands for a natural number or (countable) infinite, and $E_{j}(\cdot)$ is the resolution of identity of $T_{j}$. For each fixed $\psi \in \mathcal{H}$ with $\|\psi\|=1$, the support of the spectral measure $\mu_{\psi}^{T}(\cdot):=$ $\langle\psi, E(\cdot) \psi\rangle$, denoted by $\operatorname{supp}\left(\mu_{\psi}^{T}\right)$, is the intersection of all closed subsets of $\mathbb{R}^{N}$ with full $\mu_{\psi}^{T}$ measure $\left(\mathbb{R}^{\mathbb{N}}\right.$ with the product topology). We also set $J_{N}=\{1,2, \cdots, N\}$ if $N \in \mathbb{N}$, and $J_{N}=\mathbb{N}$ in case $N=\infty$.

Definition 1.5 Let $K$ denote either $H$ or $P$, for Hausdorff or packing, respectively. Let $\mu$ be a probability product-measure on the Borel sets $\left(\mathbb{R}^{N} ; \mathcal{B}\left(\mathbb{R}^{N}\right)\right)$ given by $\mu(\cdot)=\prod_{n=1}^{N} \mu_{n}(\cdot)$. Let $I=$ $\prod_{n=1}^{N} I_{n} \in \mathcal{B}\left(\mathbb{R}^{N}\right)$ be a measurable rectangle. One says that $\operatorname{dim}_{\mathrm{K}}^{ \pm}(\mu)$ is minimal if, for each $n \in J_{N}$, $\operatorname{dim}_{\mathrm{K}}^{ \pm}\left(\mu_{n}\right)=0$. Accordingly, one says that $\operatorname{dim}_{\mathrm{K}}^{ \pm}(\mu)$ is maximal if, for each $n \in J_{N}, \operatorname{dim}_{\mathrm{K}}^{ \pm}\left(\mu^{n}\right)=n$, where

$$
\mu^{n}:=\prod_{k=1}^{n} \mu_{k}
$$

Denote by $X$ the collection of such families of countable sequences of pairwise commuting selfadjoint operators, and let $d$ be any metric in $X$ whose convergence implies, for each $k \in J_{N}$, strong resolvent convergence; one could set, for instance,

$$
d\left(T, T^{\prime}\right):=\sup _{k \in J_{N}} D\left(T_{k}, T_{k}^{\prime}\right),
$$

where

$$
D\left(T_{k}, T_{k}^{\prime}\right):=\sum_{l \geq 1} \min \left(2^{-l},\left\|\left(T_{k}-T_{k}^{\prime}\right) \xi_{l}\right\|\right)
$$


$\left((\xi)_{l \geq 1}\right.$ is an orthonormal basis of $\left.\mathcal{H}\right)$. Naturally, $(X, d)$ is a complete metric space. The following result is the natural extension of Theorem 1.1 to this setting.

Theorem 1.6 Let $\psi \in \mathcal{H}$, with $\|\psi\|=1$, let for each $j \in J_{N}, \emptyset \neq F_{j}$ be a closed set and put $F:=\prod_{j=1}^{J_{N}} F_{j}$. Suppose that each of the sets

- $C_{\min }^{\psi ; F}=\left\{T \in X \mid \operatorname{dim}_{\mathrm{H}}^{+}\left(\mu_{\psi ; F}^{T}\right)\right.$ is minimal $\}$,

- $C_{\max }^{\psi ; F}=\left\{T \in X \mid \operatorname{dim}_{\mathrm{P}}^{-}\left(\mu_{\psi ; F}^{T}\right)\right.$ is maximal $\}$,

is dense in $X$. Then, the set $\left\{T \in X \mid \operatorname{dim}_{\mathrm{H}}^{+}\left(\mu_{\psi ; F}^{T}\right)\right.$ is minimal and $\operatorname{dim}_{\mathrm{P}}^{-}\left(\mu_{\psi ; F}^{T}\right)$ is maximal $\}$ is generic in $X$.

One can prove Theorem 1.6 using adapted versions of the results stated in Section 3 for functions defined in $\mathbb{R}^{n}$, with $n \in J_{N}$.

The result stated in Theorem 1.6 is particularly true for the set of normal operators acting in $\mathcal{H}$, which we denote by $Y$; recall that a normal operator $A$ can be written in terms of a pair $T_{1}, T_{2}$ of commuting self-adjoint operators: $A=f\left(T_{1}, T_{2}\right)$, where $f: \mathbb{R}^{2} \rightarrow \mathbb{C}, f\left(x_{1}, x_{2}\right)=x_{1}+i x_{2}$. This also leads to a version of Simon's Wonderland Theorem [15] to normal operators.

Theorem 1.7 Let $(Y, d)$ be as above, and suppose that each of the sets

- $\{A \in Y \mid A$ has purely absolutely continuous spectrum $\}$,

- $\{A \in Y \mid A$ has pure point spectrum $\}$

is dense in $Y$. Then, the set $\{A \in Y \mid A$ has purely singular continuous spectrum $\}$ is generic in $Y$.

In what follows, we use the remark above in order to extend the result stated in Theorem 3.1 in [15] to normal operators. Let $a:=\left(a_{1}, a_{2}\right)$ be such that $a_{1}, a_{2}>0$, and set $Y^{a}=\left\{A \in Y \mid\left\|T_{1}\right\| \leq a_{1}\right.$, $\left.\left\|T_{2}\right\| \leq a_{2}\right\}$.

Theorem 1.8 Let $\psi \in \mathcal{H}$ with $\|\psi\|=1$ and set $R:=\left[-a_{1}, a_{1}\right] \times\left[-a_{2}, a_{2}\right]$. Then, the set $\left\{A \in Y^{b} \mid\right.$ $\operatorname{supp}\left(\mu_{\psi}^{A}\right)=R, \operatorname{dim}_{\mathrm{H}}^{+}\left(\mu_{\psi}^{A}\right)$ is minimal, $\operatorname{dim}_{\mathrm{P}}^{-}\left(\mu_{\psi}^{A}\right)$ is maximal\} is generic in $Y^{a}$.

\subsection{Organization}

In Section 2 we recall important decompositions of Borel measures on $\mathbb{R}$ with respect to Hausdorff and packing dimensions, along with the corresponding spectral decompositions of self-adjoint operators. Section 3 is dedicated to the construction of suitable $G_{\delta}$ sets. In Section 4 we present the proofs of Theorems 1.1 and 1.4

Now some words about notation. $\mathcal{H}$ will always denote a complex separable Hilbert space. $\mathcal{B}(\mathbb{R})$ denotes the collection of Borel sets in $\mathbb{R} ; \mu$ will always indicate a finite nonnegative Borel measure on $\mathbb{R}$, and its restriction to the Borel set $A$ will be indicated by $\mu_{; A}(\cdot):=\mu(A \cap \cdot)$. The adjective absolutely continuous without specification means that $\mu$ is absolutely continuous with respect to Lebesgue measure on $\mathbb{R}$. A nonnegative Borel measure $\nu$ on $\mathbb{R}$ is supported on a Borel set $S$ if $\nu\left(\mathbb{R}^{n} \backslash S\right)=0$. Finally, it will also be convenient to use the symbol $\mathrm{K}$ to refer to either $\mathrm{H}$ or $\mathrm{P}$, which stands for Hausdorff and packing properties, respectively. 


\section{Preliminaries}

\subsection{Hausdorff and packing measures}

Let us recall the definitions of Hausdorff and packing measures on $\mathbb{R}$.

Definition 2.9 Let $A \subset \mathbb{R}$. By a $\delta$-covering of $A$ we mean any countable collection $\left\{E_{k}\right\}$ of subsets of $\mathbb{R}$ such that $A \subset \cup_{k \geq 1} E_{k}$ and $\operatorname{diam}\left(E_{k}\right):=\sup _{x, y \in E_{k}}|x-y| \leq \delta$. For each $\alpha \in[0,1]$, the $\alpha$ dimensional (exterior) Hausdorff measure of $A$ is defined as

$$
h^{\alpha}(S)=\liminf _{\delta \downarrow 0}\left\{\sum_{k=1}^{\infty} \operatorname{diam}\left(E_{k}\right)^{\alpha} \mid\left\{E_{k}\right\} \text { is a } \delta \text {-covering of } S\right\} .
$$

The Hausdorff dimension of the set $S$, here denoted by $\operatorname{dim}_{\mathrm{H}}(S)$, is defined as the infimum of all $\alpha$ such that $h^{\alpha}(S)=0$; note that $h^{\alpha}(S)=\infty$ if $\alpha<\operatorname{dim}_{\mathrm{H}}(S)$.

A $\delta$-packing of an arbitrary set $S \subset \mathbb{R}$ is a countable disjoint collection $\left(\bar{B}\left(x_{k} ; r_{k}\right)\right)_{k \in \mathbb{N}}$ of closed balls centered at $x_{k} \in S$ and radii $r_{k} \leq \delta / 2$, so with diameters at most of $\delta$. Define $P_{\delta}^{\alpha}(S), \alpha \in[0,1]$, as

$$
P_{\delta}^{\alpha}(S)=\sup \left\{\sum_{k=1}^{\infty}\left(2 r_{k}\right)^{\alpha} \mid\left(\bar{B}\left(x_{k} ; r_{k}\right)\right)_{k} \text { is a } \delta \text {-packing of } A\right\},
$$

that is, the supremum is taken over all $\delta$-packings of $S$. Then, take the decreasing limit

$$
P_{0}^{\alpha}(S)=\lim _{\delta \downarrow 0} P_{\delta}^{\alpha}(S)
$$

which is a pre-measure.

Definition 2.10 The $\alpha$-packing (exterior) measure $P^{\alpha}(S)$ of $S$ is given by

$$
P^{\alpha}(S):=\inf \left\{\sum_{k=1}^{\infty} P_{0}^{\alpha}\left(E_{k}\right) \mid S \subset \bigcup_{k=1}^{\infty} E_{k}\right\} .
$$

The packing dimension of the set $S$, here denoted by $\operatorname{dim}_{\mathrm{P}}(S)$, is defined (in analogy to $\operatorname{dim}_{\mathrm{H}}(S)$ ) as the infimum of all $\alpha$ such that $P^{\alpha}(S)=0$, which coincides with the supremum of all $\alpha$ so that $P^{\alpha}(S)=\infty$.

It is known [12 that $\operatorname{dim}_{\mathrm{H}}(S) \leq \operatorname{dim}_{\mathrm{P}}(S)$, and this inequality is in general strict. It is also important to mention that $P^{\alpha}$ and $h^{\alpha}$ are Borel (regular) measures; furthermore, $P^{0} \equiv h^{0}, P^{1} \equiv h^{1}$, and they are equivalent, respectively, to the counting measure (which assigns to each set $S$ the number of elements it has) and the Lebesgue measure.

Definition 2.11 Let $\alpha \in[0,1]$. A finite nonnegative Borel measure $\mu$ on $\mathbb{R}$ is called:

1. $\alpha-\mathrm{K}$ continuous, denoted $\alpha \mathrm{Kc}$, if $\mu(S)=0$ for every Borel set $S$ such that $\mathrm{K}^{\alpha}(S)=0$.

2. $\alpha-\mathrm{K}$ singular, denoted $\alpha \mathrm{Ks}$, if it is supported on some Borel set $S$ with $\mathrm{K}^{\alpha}(S)=0$. 
3. 0-K dimensional, denoted $0 \mathrm{Kd}$, if it is supported on a Borel set $S$ with $\operatorname{dim}_{\mathrm{K}}(S)=0$.

4. $1-\mathrm{K}$ dimensional, denoted $1 \mathrm{Kd}$, if $\mu(S)=0$ for any Borel set $S$ with $\operatorname{dim}_{\mathrm{K}}(S)<1$.

Remark 2.12 1. $\mu$ is $0 \mathrm{Kd}$ if, and only if, it is $\alpha \mathrm{Ks}$ for each $\alpha \in(0,1]$. Equivalently, $\mu$ is $1 \mathrm{Kd}$ if, and only if, it is $\alpha \mathrm{Kc}$ for each $\alpha \in[0,1)$.

2. It follows from Definition 2.11 that $\mu$ is $0 \mathrm{Kd}$ if it is pure point, whereas $\mu$ is $1 \mathrm{Kd}$ if it is absolutely continuous.

Definition 2.13 Let $\mu$ be a finite nonnegative Borel measure on $\mathbb{R}$ and $x \in \mathbb{R}$. Set $B(x ; \varepsilon)=\{y \in$ $\mathbb{R}|| x-y \mid<\varepsilon\}$, i.e., the open ball of radius $\varepsilon>0$ centered at $x$, and

$$
D_{\mu}^{\mathrm{H}, \alpha}(x):=\limsup _{\varepsilon \downarrow 0} \frac{\mu(B(x ; \varepsilon))}{(2 \varepsilon)^{\alpha}}, \quad D_{\mu}^{\mathrm{P}, \alpha}(x):=\liminf _{\varepsilon \downarrow 0} \frac{\mu(B(x ; \varepsilon))}{(2 \varepsilon)^{\alpha}} .
$$

The following density results [9, 14] relate the continuity of $\mu$, with respect to Hausdorff (packing) dimension, to its local scaling behavior as probed by $D_{\mu}^{\mathrm{K}, \alpha}$.

Theorem 2.14 Let $\mu$ be as above and let $\alpha \in[0,1]$. Let

$$
K_{\alpha \mathrm{Kc}}:=\left\{x \in \mathbb{R} \mid D_{\mu}^{\mathrm{K}, \alpha}(x)<\infty\right\}, \quad K_{\alpha \mathrm{Ks}}:=\left\{x \in \mathbb{R} \mid D_{\mu}^{\mathrm{K}, \alpha}(x)=\infty\right\} .
$$

Then, these are Borel sets, $\mu_{\alpha \mathrm{Kc}}(\cdot):=\mu\left(K_{\alpha \mathrm{Kc}} \cap \cdot\right)$ is $\alpha \mathrm{Kc}, \mu_{\alpha \mathrm{Ks}}(\cdot):=\mu\left(K_{\alpha \mathrm{Ks}} \cap \cdot\right)$ is $\alpha \mathrm{Ks}, \mu_{0 \mathrm{Kd}}(\cdot):=$ $\mu\left(\left(\bigcap_{k \geq 1} K_{(1 / \mathrm{k}) \mathrm{Ks}}\right) \cap \cdot\right)$ is $0 \mathrm{Kd}$, and $\mu_{1 \mathrm{Kd}}(\cdot):=\mu\left(\left(\bigcap_{k \geq 1} K_{(1-1 / \mathrm{k}) \mathrm{Kc}}\right) \cap \cdot\right)$ is $1 \mathrm{Kd}$.

Proof. See Section 4 in [1] for the Hausdorff case; the packing case follows analogously.

By following [9, 16, we recall the upper and lower dimensions of a finite Borel measure $\mu$.

Definition 2.15 Let $\mu$ be as above, and let $I \subset \mathbb{R}$ be a Borel set. The $\mathrm{K}$ upper dimension of $\mu$ restricted to $I$, denoted by $\operatorname{dim}_{\mathrm{K}}^{+}\left(\mu_{; I}\right)$, is defined as

$$
\operatorname{dim}_{\mathrm{K}}^{+}\left(\mu_{; I}\right):=\inf \left\{\operatorname{dim}_{\mathrm{K}}(S) \mid \mu(I \backslash S)=0, S \text { a Borel subset of } I\right\},
$$

and the $\mathrm{K}$ lower dimension of $\mu$ restricted to $I$, denoted by $\operatorname{dim}_{\mathrm{K}}^{-}\left(\mu_{; I}\right)$, as

$$
\operatorname{dim}_{\mathrm{K}}^{-}\left(\mu_{; I}\right):=\sup \left\{\alpha \mid \mu(S)=0 \text { if } \operatorname{dim}_{\mathrm{K}}(S)<\alpha, S \text { a Borel subset of } I\right\} \text {. }
$$

When $I=\mathbb{R}$, we simply denote $\operatorname{dim}_{\mathrm{K}}^{ \pm}\left(\mu_{; I}\right)$ by $\operatorname{dim}_{\mathrm{K}}^{ \pm}(\mu)$.

Proposition 2.16 Let $\mu$ be as above, let I be a Borel subset of $\mathbb{R}$, and let $\alpha \in(0,1)$. Then,

1. $\alpha \leq \operatorname{dim}_{\mathrm{K}}^{-}\left(\mu_{; I}\right)$ if, and only if, for each $\varepsilon \in(0, \alpha], \mu_{; I}$ is $(\alpha-\varepsilon) \mathrm{Kc}$;

2. $\operatorname{dim}_{\mathrm{K}}^{+}\left(\mu_{; I}\right) \leq \alpha$ if, and only if, for each $\varepsilon \in(0,1-\alpha], \mu_{; I}$ is $(\alpha+\varepsilon) \mathrm{Ks}$.

Proof. See Section 1 in [2]. 


\section{$3 G_{\delta}$ sets}

Let $(X, d)$ be as in the Introduction, let $\emptyset \neq O \subset \mathbb{R}$ be an open set, and let

$$
\mathcal{M}_{+}(O):=\{\mu \in \mathcal{M}(O) \mid 0 \leq \mu \leq 1\}
$$

that is, the set of positive measures on $O$ with total mass less than or equal to one. We endow such set with the weak topology, i.e., the topology of the weak convergence of measures $\left(\left(\mu_{n}\right)\right.$ converges weakly to $\mu$ if for each $f \in C_{b}(O), \int f(x) d \mu_{n}(x) \rightarrow \int f(x) d \mu(x)$; here, $C_{b}(O)$ denotes the set of bounded continuous functions defined on $O$ ). Recall that such topology is metrizable (since $O$ is a Polish space): take, for instance, the Lévy-Prohorov metric, which will be denoted by $\rho$ (see Appendix 2 in [7] for details).

Let also, for each $T \in X$ and each $0 \neq \psi \in \mathcal{H}, \zeta_{\psi}: X \rightarrow \mathcal{M}_{+}(O)$ be defined by the law $\zeta_{\psi}(T):=$ $\mu_{\psi ; O}^{T}$, where $\mu_{\psi ; O}^{T}(\cdot):=\mu_{\psi}^{T}(O \cap \cdot)$. It follows from the functional calculus for self-adjoint operators that $\zeta_{\psi}$ is a continuous function: if $\lim _{m \rightarrow \infty} d\left(T_{m}, T\right)=0$, then $\lim _{m \rightarrow \infty} \rho\left(\mu_{\psi ; O}^{T_{m}}, \mu_{\psi ; O}^{T}\right)=0$.

Lemma 3.17 Let $\emptyset \neq O \subset \mathbb{R}$ be an open set and let, for each $t>0, V_{t}(\cdot, \cdot): \mathcal{M}_{+}(O) \times O \rightarrow[0,1]$ be defined by the law $V_{t}(\mu, x):=\int f_{t, x}(y) d \mu(y)$, where $f_{t, x}: O \rightarrow[0,1]$ is given by

$$
f_{t, x}(y):=\left\{\begin{array}{llc}
1 & \text {, if } & |x-y| \leq 1 / t \\
-t|x-y|+2 & \text {, if } & 1 / t \leq|x-y| \leq 2 / t \\
0 & \text {,if } & |x-y| \geq 2 / t
\end{array}\right.
$$

Let also, for each $0 \neq \psi \in \mathcal{H}, U_{t, \psi}(\cdot, \cdot): X \times O \rightarrow[0,1]$ be defined by the law

$$
U_{t, \psi}(T, x):=\left(\psi, f_{t, x}(T) \psi\right)=\int f_{t, x}(y) d \mu_{\psi ; O}^{T}(y) .
$$

Then, $U_{t, \psi}(T, x)=V_{t}\left(\zeta_{\psi}(T), x\right)$ and

$$
\left(D^{\mathrm{K}, \alpha} \mu_{\psi ; O}^{T}\right)(x)=\lim _{t \rightarrow \infty} \mathrm{K} t^{\alpha} U_{t, \psi}(T, x) .
$$

Furthermore, for each $t>0$, the function $V_{t}: \mathcal{M}_{+}(O) \times O \rightarrow[0,1]$ is jointly continuous.

Proof. It follows from the the Spectral Theorem that, for each $x \in O$, each $t>0$ and each $0 \neq \psi \in \mathcal{H}$,

$$
\mu_{\psi ; O}^{T}\left(B_{1 / t}(x)\right) \leq U_{t, \psi}(T, x)=\int f_{t, x}(y) d \mu_{\psi ; O}^{T}(y) \leq \mu_{\psi ; O}^{T}\left(B_{2 / t}(x)\right) .
$$

Then, one has $t^{\alpha} \mu_{\psi ; O}^{T}(B(x, 1 / t)) \leq t^{\alpha} U_{t, \psi}(T, x) \leq t^{\alpha} \mu_{\psi ; O}^{T}(B(x, 2 / t))$, which proves the first assertion.

Note that, for each $x \in O$ and each $t>0, f_{t, x}: O \rightarrow \mathbb{R}$ is a continuous function such that, for each $y \in O, \chi_{B(x, 1 / t)}(y) \leq f_{t, x}(y) \leq \chi_{B(x, 2 / t)}(y)$. Given that each $f_{t, x}(y)$ depends only on $|x-y|$, it is straightforward to show that for each $t>0, f_{t, x_{l}}$ converges uniformly to $f_{t, x}$ on $O$ when $x_{l} \rightarrow x$.

We combine this remark with Theorems 2.13 and 2.15 in [10] in order to prove that $V_{t}(\mu, x)$ is jointly continuous. Let $\left(\mu_{m}\right)$ and $\left(x_{l}\right)$ be sequences in $\mathcal{M}_{+}(O)$ and $O$, respectively, such that $\rho\left(\mu_{m}, \mu\right) \rightarrow 0$ and $x_{l} \rightarrow x$. Firstly, we show that

$$
\lim _{m \rightarrow \infty} \lim _{l \rightarrow \infty} U_{t, \psi}\left(\mu_{m}, x_{l}\right)=\lim _{m \rightarrow \infty} \lim _{l \rightarrow \infty} \int f_{t, \psi}(y) d \mu_{m}(y)=V_{t}(\mu, x) .
$$


Since, for each $y \in \mathbb{R},\left|f_{t, x_{l}}(y)\right| \leq 1$, it follows from dominated convergence that, for each $m \in \mathbb{N}$, $\lim _{l \rightarrow \infty} \int f_{t, x_{l}}(y) d \mu_{m}(y)=\int f_{t, x}(y) d \mu_{m}(y)$. Now, since $f_{t, x}$ is continuous and convergence in the metric $\rho$ implies weak convergence of measures, one has

$$
\lim _{m \rightarrow \infty} \lim _{l \rightarrow \infty} \int f_{t, x_{l}}(y) d \mu_{m}(y)=\lim _{m \rightarrow \infty} \int f_{t, x}(y) d \mu_{m}(y)=V_{t}(\mu, x) .
$$

The next step consists in showing that, for each $l \in \mathbb{N}$, the function $\varphi_{l}: \mathbb{N} \rightarrow \mathbb{R}$, defined by the law $\varphi_{l}(m):=V_{t}\left(\mu_{m}, x_{l}\right)$, converges uniformly to $\varphi(m):=\lim _{l \rightarrow \infty} V_{t}\left(\mu_{m}, x_{l}\right)=\int f_{t, x}(y) d \mu_{m}(y)$. Let $\delta>0$. Since, for each $t>0, f_{t, x_{l}}(y)$ converges uniformly to $f_{t, x}(y)$, there exists $N \in \mathbb{N}$ such that, for each $l \geq N$ and each $y \in \mathbb{R},\left|f_{t, x_{l}}(y)-f_{t, x}(y)\right|<\delta$. Then, one has, for each $l \geq N$ and each $m \in \mathbb{N}$,

$$
\begin{aligned}
\left|\varphi_{l}(m)-\varphi(m)\right| & =\left|\int f_{t, x_{l}}(y) d \mu_{m}(y)-\int f_{t, x}(y) d \mu_{m}(y)\right| \\
& \leq \int\left|f_{t, x_{l}}(y)-f_{t, x}(y)\right| d \mu_{m}(y)<\delta .
\end{aligned}
$$

It follows from Theorem 2.15 in [10] that $\lim _{l, m \rightarrow \infty} V_{t}\left(\mu_{m}, x_{l}\right)=V_{t}(\mu, x)$. Given that $\lim _{l \rightarrow \infty} V_{t}\left(\mu_{m}, x_{l}\right)=\int f_{t, x}(y) d \mu_{m}(y)$ and that $\lim _{m \rightarrow \infty} V_{t}\left(\mu_{m}, x_{l}\right)=\int f_{t, x_{l}}(y) d \mu(y)$ exist for each $m \in \mathbb{N}$ and each $l \in \mathbb{N}$, respectively, Theorem 2.13 in [10] implies that

$$
\lim _{m \rightarrow \infty} \lim _{l \rightarrow \infty} V_{t}\left(\mu_{m}, x_{l}\right)=\lim _{l \rightarrow \infty} \lim _{m \rightarrow \infty} V_{t}\left(\mu_{m}, x_{l}\right)=\lim _{l, m \rightarrow \infty} V_{t}\left(\mu_{m}, x_{l}\right)=V_{t}(\mu, x) .
$$

Hence, if $\left(\mu_{l}, x_{l}\right)$ is some sequence in $\mathcal{M}_{+}(O) \times O$ (endowed with the product topology) such that $\left(\mu_{l}, x_{l}\right) \rightarrow(\mu, x) \in \mathcal{M}_{+}(O) \times O$, then $\lim _{l \rightarrow \infty} V_{t}\left(\mu_{l}, x_{l}\right)=V_{t}(\mu, x)$, showing that $V_{t}(\cdot, \cdot)$ is jointly continuous at $(\mu, x)$.

Before we present our main result, some preparation is required. Let, for each $\alpha \in(0,1), \beta_{\mu}^{\mathrm{H}, \alpha}$ : $E \times \mathbb{N} \rightarrow[0,+\infty)$ be defined by the law $\beta_{\mu}^{\mathrm{H}, \alpha}(x, s):=\sup _{t \geq s} t^{\alpha} V_{t}(\mu, x)$, where for each $t>0$, $V_{t}(\cdot, \cdot): \mathcal{M}_{+}(E) \times E$ is defined as in the statement of Lemma 3.17

Remark 3.18 The proof that, for each $t>0$, the mapping $V_{t}(\cdot, \cdot): \mathcal{M}_{+}(E) \times E, V_{t}(\mu, x)=$ $\int f_{t, x}(y) d \mu(y)$, is jointly continuous if $(E, d)$ is a Polish metric space is identical to the proof of Lemma 3.17 in the definition of $f_{t, x}$, just replace the euclidean metric in $\mathbb{R}$ by $d$.

Lemma 3.19 Let $E$ be a Polish metric space and let $\alpha \in(0,1)$. Then, for each $\delta>0$ and each $r, s \in \mathbb{N}$,

$$
\mathcal{M}_{r, s}(\delta):=\left\{\mu \in \mathcal{M}_{+}(E) \mid \mu\left(Z_{\mu}(r, s)\right) \geq \delta\right\}
$$

is a closed subset of $\mathcal{M}(E)$, where $Z_{\mu}(r, s):=\left\{x \in E \mid \beta_{\mu}^{\mathrm{H}, \alpha}(x, s) \leq r\right\}$.

Proof. Claim 1. For each $r, s \in \mathbb{N}$ and each $\mu \in \mathcal{M}_{+}(E), Z_{\mu}(r, s)$ is a closed subset of $E$.

Let $\left\{w_{i}\right\}$ be a sequence in $Z_{\mu}(r, s)$ such that $\lim w_{i}=w$. Since, for each $t>0, f_{t, w_{i}} \rightarrow f_{t, w}$ pointwise, it follows from Remark 3.18 that the mapping $x \mapsto \beta_{\mu}^{\mathrm{H}, \alpha}(x, s)$ is lower semi-continuous. Hence, $\beta_{\mu}^{\mathrm{H}, \alpha}(w, s) \leq r$, which means that $w \in Z_{\mu}(r, s)$. 
Claim 2. For each $s \in \mathbb{N}, W_{r, s}=\left\{(\nu, x) \in \mathcal{M}(E) \times E \mid \beta_{\nu}^{H, \alpha}(x, s)>r\right\}$ is open.

This is a consequence of the fact that, by Remark 3.18, the mapping $\mathcal{M}_{+}(E) \times E \ni(\nu, x) \longmapsto$ $\beta_{\nu}^{H, \alpha}(x, s)$ is lower semi-continuous.

Now, we show that $\mathcal{M}_{r, s}(\delta)$ is closed. Let $\mu_{m}$ be a sequence in $\mathcal{M}_{r, s}(\delta)$ such that $\mu_{m} \rightarrow \mu$. Suppose, by absurd, that $\mu \notin \mathcal{M}_{r, s}(\delta)$; we will find that $\mu_{m} \notin \mathcal{M}_{r, s}(\delta)$ for $m$ sufficiently large, a contradiction.

If $\mu \notin \mathcal{M}_{r, s}(\delta)$, then $\mu(A)>\mu(E)-\delta$, where $A:=E \backslash Z_{\mu}(r, s)$. Hence $\{\mu\} \times A \subset W_{r}$. Since $\mu$ is a tight measure on $E$ ( $\mu$ is a Borel measure and the space $X$ is Polish; see Proposition A.2.2.V in [7]), there exists a compact $C \subset A$ such that $\mu(C)>\mu(E)-\delta$ (note that, by Claim $1, A$ is open).

Now, we construct a suitable subset of $W_{r, s}$ that contains a neighborhood of $\{\mu\} \times C$. Let, for each $x \in C, V_{x} \subset W_{r, s}$ be an open neighborhood of $(\mu, x)$ (such open set exists, by Claim 2); that is, $V_{x}:=B((\mu, x) ; \varepsilon)=\left\{(\nu, y) \in \mathcal{M}_{+}(E) \times E \mid \max \{\rho(\nu, \mu), d(x, y)\}<\varepsilon\right\}$, for some suitable $\varepsilon>0$. Then, $\left\{V_{x}\right\}_{x \in C}$ is an open cover of $\{\mu\} \times C$, and since $\{\mu\} \times C$ is a compact subset of $\mathcal{M}_{+}(E) \times E$, it follows that one can extract from $\left\{V_{x}\right\}_{x \in C}$ a finite subcover, $\left\{V_{x_{i}}\right\}_{i=1}^{n}$.

We affirm that there exists an $\ell \in \mathbb{N}$ (which depends on $C$ ) such that $\left\{\mu_{n}\right\}_{n \geq \ell} \subset \bigcap_{i}\left(\pi_{1}\left(V_{x_{i}}\right)\right.$ ). Namely, for each $i$, there exists an $\ell_{i}$ such that $\left\{\mu_{n}\right\}_{n \geq \ell_{i}} \subset \pi_{1}\left(V_{x_{i}}\right)$; set $\ell:=\max \left\{\ell_{i} \mid i \in\{1, \ldots, n\}\right\}$, and note that for each $i,\left\{\mu_{n}\right\}_{n \geq \ell} \subset \pi_{1}\left(V_{x_{i}}\right)$. Set also $\mathcal{I}:=\bigcap_{i}\left(\pi_{1}\left(V_{x_{i}}\right)\right)$ and $\mathcal{O}:=\bigcup_{i}\left(\pi_{2}\left(V_{x_{i}}\right)\right)$.

Since for each $i, V_{x_{i}}=\pi_{1}\left(V_{x_{i}}\right) \times \pi_{2}\left(V_{x_{i}}\right)$, and given that

$$
\left\{\mu_{n}\right\}_{n \geq \ell} \times \mathcal{O} \subset \mathcal{I} \times \mathcal{O} \subset \bigcup_{i}\left(\pi_{1}\left(V_{x_{i}}\right) \times \pi_{2}\left(V_{x_{i}}\right)\right)=\bigcup_{i} V_{x_{i}} \subset W_{r, s}
$$

it follows that, for each $n \geq \ell$ and each $y \in \mathcal{O}, \beta_{\mu_{m}}^{\mathrm{H}, \alpha}(y, s)>r$. Moreover, $\mathcal{O}$ is an open set that contains $C$.

On the other hand, weak convergence implies that

$$
\limsup _{m \rightarrow \infty} \mu_{m}(E \backslash \mathcal{O}) \leq \mu(E \backslash \mathcal{O}) \leq \mu(E \backslash C)<\delta,
$$

from which follows that there exists an $\ell_{1} \geq \ell$ such that, for $m \geq \ell_{1}, \mu_{m}(E \backslash \mathcal{O})<\delta$.

Combining the last results, one concludes that, for $m \geq \ell_{1}, \mu_{m}(E \backslash \mathcal{O})<\delta$, and for each $x \in \mathcal{O}$, $\beta_{\mu_{m}}^{\mathrm{H}, \alpha}(x, s)>r$, so

$$
\mu_{m}\left(Z_{\mu_{m}}(r, s)\right) \leq \mu_{m}(E \backslash \mathcal{O})<\delta
$$

this contradicts the fact that, for each $m \in \mathbb{N}, \mu_{m} \in \mathcal{M}_{r, s}(\delta)$. Hence, $\mu \in \mathcal{M}_{r, s}(\delta)$, and $\mathcal{M}_{r, s}(\delta)$ is a closed subset of $\mathcal{M}_{+}(E)$.

Define, for $\alpha \in(0,1)$ and $s \in \mathbb{N}, \gamma_{\psi, T}^{\mathrm{H}(\mathrm{P}), \alpha}(x, s):=\sup (\mathrm{inf})_{t \geq s} t^{\alpha} U_{t, \psi}(T, x)$. Then, by Lemma 3.17. one has, for each $x \in O, \lim _{s \rightarrow \infty} \gamma_{\psi, T}^{\mathrm{K}, \alpha}(x, s)=\left(D^{\mathrm{K}, \alpha} \mu_{\psi ; O}^{T}\right)(x)$. By definition, for each $x \in O$, $\mathbb{N} \ni s \mapsto \gamma_{\psi, T}^{\mathrm{H}(\mathrm{P}), \alpha}(x, s) \in[0,+\infty)$ is a nonincreasing (nondecreasing) mapping.

Theorem 3.20 Let $\emptyset \neq F \subset \mathbb{R}$ be a closed subset, let $0 \neq \psi \in \mathcal{H}$, and $\mu_{\psi ; F}^{T}(\cdot):=\mu_{\psi}^{T}(F \cap \cdot)$. Then, each of the sets $C_{0 \mathrm{Hd}}^{\psi ; F}:=\left\{T \in X \mid \operatorname{dim}_{\mathrm{H}}^{+}\left(\mu_{\psi ; F}^{T}\right)=0\right\}$ and $C_{1 \mathrm{Pd}}^{\psi ; F}:=\left\{T \in X \mid \operatorname{dim}_{\mathrm{P}}^{-}\left(\mu_{\psi ; F}^{T}\right)=1\right\}$ is a $G_{\delta}$ set in $X$. 
Proof. Since the arguments in both proofs are analogous, we just prove the statement for $C_{0 \mathrm{Hd}}^{\psi ; F}$. Note that for each closed set $F$, there exists a countable family of open sets, $\left\{A_{i}\right\}$, such that $F=\bigcap_{i>1} A_{i}$ (each closed set $F$ is a $G_{\delta}$ set); thus, one just has to prove the result for $C_{0 \mathrm{Hd}}^{\psi ; O}$, where $\emptyset \neq O \subset \mathbb{R}$ is an open set.

If, for each $T \in X, \mu_{\psi ; O}^{T}(\mathbb{R})=0$, then $C_{0 \mathrm{Hd}}^{\psi ; O}=\emptyset$ is a $G_{\delta}$ subset of $X$. Thus, suppose that $\left\{T \in X \mid \mu_{\psi ; O}^{T}(\mathbb{R})>0\right\} \neq \emptyset$.

Set, for each $\alpha \in(0,1), C_{\alpha \mathrm{Hc}}^{\psi ; O}:=\bigcup_{p \geq 1} C_{\alpha \mathrm{Hc}}^{\psi ; O}(p)$, where $C_{\alpha \mathrm{Hc}}^{\psi ; O}(p)=\left\{T \in X \mid \mu_{\psi ; O}^{T}(\{x \in \mathbb{R} \mid\right.$ $\left.\left.\left.\left(D^{\mathrm{H}, \alpha} \mu_{\psi ; O}^{T}\right)(x)<p\right\}\right)>0\right\}$. Now, by Theorem 2.14 and Proposition 2.16.

$$
C_{0 \mathrm{Hd}}^{\psi ; O}=\bigcap_{k>1}\left(C_{(1 / \mathrm{k}) \mathrm{Hc}}^{\psi ; O}\right)^{c}=\bigcap_{k>1} \bigcap_{p \geq 1}\left(C_{(1 / \mathrm{k}) \mathrm{Hc}}^{\psi ; O}(p)\right)^{c} .
$$

Claim 1. For each $\alpha \in(0,1)$ and each $p \in \mathbb{N}$,

$$
\left(C_{\alpha \mathrm{Hc}}^{\psi ; O}(p)\right)^{c}=\bigcap_{s \in \mathbb{N}}\left\{T \in X \mid \mu_{\psi ; O^{-}}^{T} \text { ess. inf } \gamma_{\psi, T}^{\mathrm{H}, \alpha}(x, s) \geq p\right\} .
$$

Let $T \in\left(C_{\alpha \mathrm{Hc}}^{\psi ; O}(p)\right)^{c}$. Since, for each $x \in \mathbb{R}, \mathbb{N} \ni s \mapsto \gamma_{\psi, T}^{\mathrm{H}, \alpha}(x, s) \in[0,+\infty)$ is a nonincreasing function, it follows that, for each $s \in \mathbb{N}, \mu_{\psi ; O^{-}}^{T}$ ess. inf $\gamma_{\psi, T}^{\mathrm{H}, \alpha}(x, s) \geq p$.

Now, let $T \in \bigcap_{s \in \mathbb{N}}\left\{U \in X \mid \mu_{\psi ; O^{-}}^{U}\right.$ ess. inf $\left.\gamma_{\psi, U}^{\mathrm{H}, \alpha}(x, s) \geq p\right\}$. Then, for each $s \in \mathbb{N}$, there exits a Borel set $A_{s} \subset \mathbb{R}$, with $\mu_{\psi ; O}^{T}\left(A_{s}\right)=1$, such that for each $x \in A_{s}, \gamma_{\psi, T}^{\mathrm{H}, \alpha}(x, s) \geq p$. Let $A:=\bigcap_{s \geq 1} A_{s}$; then, for each $x \in A$, one has $\left(D^{\mathrm{H}, \alpha} \mu_{\psi ; O}^{T}\right)(x)=\lim _{s \rightarrow \infty} \gamma_{\psi, U}^{\mathrm{H}, \alpha}(x, s) \geq p$; given that $\mu_{\psi ; O}^{T}(A)=1$, we are done.

Let, for each $\alpha \in(0,1)$ and each $p, q, s, l \in \mathbb{N}$,

$$
C_{\alpha \mathrm{Hc}}^{\psi ; O}(p-1 / q, s, l):=\left\{T \in X \mid \mu_{\psi ; O}^{T}\left(A_{\psi ; O}^{T}(p-1 / q, s)\right) \geq 1 / l\right\}
$$

where $A_{\psi ; O}^{T}(p-1 / q, s):=\left\{x \in O \mid \gamma_{\psi, T}^{\mathrm{H}, \alpha}(x, s) \leq p-1 / q\right\}$. Thus, according to Claim 1 and (3.4),

$$
C_{0 \mathrm{Hd}}^{\psi ; O}=\bigcap_{k>1} \bigcap_{p \geq 1} \bigcap_{q \geq 1} \bigcap_{s \geq 1} \bigcap_{l \geq 1}\left(C_{(1 / \mathrm{k}) \mathrm{Hc}}^{\psi ; O}(p-1 / q, s, l)\right)^{c},
$$

and one just needs to show that, for each $\alpha \in(0,1)$ and each $r, s, l \in \mathbb{N}, C_{\alpha \mathrm{Hc}}^{\psi ; O}(r, s, l)$ is closed in $X$.

Claim 2. For each $\delta>0$ and each $r, s \in \mathbb{N},\left\{\mu \in \mathcal{M}_{+}(O) \mid \mu\left(Z_{\mu}(r, s)\right) \geq \delta\right\}$ is a closed subset of $\mathcal{M}_{+}(O)$, where $Z_{\mu}(r, s)=\left\{x \in O \mid \beta_{\mu}^{\mathrm{H}, \alpha}(x, s) \leq r\right\}$.

Here, we use the fact that $O$ can be isometrically embedded in $\bar{O}$, which is a Polish metric space. Thus, any $\mu \in \mathcal{M}_{+}(O)$ can be identified with the measure $\tilde{\mu} \in \mathcal{M}_{+}(\bar{O})$ defined by $\tilde{\mu}(A)=$ $\mu(A \cap O)$ for each $A \in \mathcal{B}(\bar{O})$, and $\mathcal{M}_{+}(O)$ can be identified with a subset of $\mathcal{M}_{+}(\bar{O})$, namely, the set $\left\{\tilde{\mu} \in \mathcal{M}_{+}(\bar{O}) \mid \tilde{\mu}(\bar{O})=\tilde{\mu}(O)\right\}$. Then, the induced topology in $\mathcal{M}_{+}(O)$ by the Polish space $\mathcal{M}_{+}(\bar{O})$ coincides with the weak topology in $\mathcal{M}_{+}(O)$ (see Section 6 in [13] for details).

Moreover, for each $\mu \in \mathcal{M}_{+}(O)$ and each $r, s \in \mathbb{N}, Z_{\mu}(r, s)=\left\{x \in \bar{O} \mid \beta_{\tilde{\mu}}^{\mathrm{H}, \alpha}(x, s) \leq r\right\} \cap O$, so for each $\delta>0$,

$$
\left\{\mu \in \mathcal{M}_{+}(O) \mid \mu\left(Z_{\mu}(r, s)\right) \geq \delta\right\}=\left\{\tilde{\mu} \in \mathcal{M}_{+}(\bar{O}) \mid \tilde{\mu}\left(Z_{\tilde{\mu}}(r, s)\right)=\mu\left(Z_{\mu}(r, s)\right) \geq \delta\right\} \cap \mathcal{M}_{+}(O) .
$$


The result is now a consequence of Lemma 3.19.

Recall that by the functional calculus, for each $0 \neq \psi \in \mathcal{H}$, the mapping $\zeta_{\psi}: X \rightarrow \mathcal{M}_{+}(O)$, $\zeta_{\psi}(T)=\mu_{\psi ; O}^{T}$ is continuous (since convergence in $X$ implies strong resolvent convergence), and note that for each $(x, s) \in O \times \mathbb{N}, \gamma_{\psi, T}^{\mathrm{H}, \alpha}(x, s)=\beta_{\zeta_{\psi}(T)}^{\mathrm{H}, \alpha}(x, s)$.

Thus, it follows that for each $l, r, s \in \mathbb{N}, C_{\alpha \mathrm{Hc}}^{\psi ; O}(r, s, l)=\left(\gamma_{\psi}\right)^{-1}\left(\mathcal{M}_{r, s}(1 / l)\right)$, and therefore, by Claim 2, $C_{\alpha \mathrm{Hc}}^{\psi ; O}(r, s, l)$ is a closed subset of $X$.

\section{Proof of Theorems 1.1 and 1.4}

Proof. (Theorem 1.1) The result is a direct consequence of the hypotheses, Theorem 3.20, and the fact that the intersection of a countable family of generic sets is still a generic set.

In order to prove Theorem 1.4 we need the following result.

Theorem 4.21 (Theorems 1.1 in [5] and 1.3 in [6]) Suppose that $\Omega$ is a Cantor group and that $\tau: \Omega \rightarrow \Omega$ is a minimal translation. Then, there exist dense sets of $g \in \mathrm{C}(\Omega, \mathbb{R})$ such that, for each $\kappa \in \Omega$,

1. the spectrum of $H_{g, \tau}^{\kappa}$ is purely absolutely continuous;

2. the spectrum of $H_{g, \tau}^{\kappa}$ is zero-Hausdorff dimensional.

Proof. (Theorem 1.4) Fix $\kappa \in \Omega$ and let $\tau: \Omega \rightarrow \Omega$ be a minimal translation of the Cantor group $\Omega$.

It follows from Theorem 4.21 that each of the sets $C_{1 \mathrm{Pd}}^{\kappa} \supset C_{\mathrm{ac}}^{\kappa}:=\left\{T \in X_{\kappa} \mid \sigma(T)\right.$ is purely absolutely continuous $\}$ and $C_{0 \mathrm{Hd}}^{\kappa}$ is dense in $X_{\kappa}$.

The result is now a consequence of Theorem 1.1 and Remark 1.3 ,

\section{Acknowledgments}

SLC thanks the partial support by FAPEMIG (Universal Project CEX-APQ-00554-13). CRdO thanks the partial support by CNPq (under contract 303503/2018-1).

\section{References}

[1] A. Avila, On the spectrum and Lyapunov exponent of limit-periodic Schrödinger operators, Commun. Math. Phys. 288 (2009), 907-918.

[2] S.L. Carvalho and C.R. de Oliveira, Correlation dimension wonderland theorems, J. Math. Phys., 57 (2016), 062902(19pp.). 
[3] S.L. Carvalho and C.R. de Oliveira, Generic quasilocalized and quasiballistic discrete Schrödinger operators, Proc. Amer. Math. Soc., 144 (2016), 129-141.

[4] S.L. Carvalho and C.R. de Oliveira, A characterization of singular packing subspaces with an application to limit-periodic operators, Forum Math. 29 (2017) 31-40 .

[5] D. Damanik and Z. Gan, Spectral properties of limit-periodic Schrödinger operators, Commun. Pure Appl. Anal. 10 (2011), 859-871.

[6] D. Damanik and Z. Gan, Limit-periodic Schrödinger operators in the regime of positive Lyapunov exponents, J. Funct. Anal. 258 (2010), 4010-4025.

[7] D. J. Daley and D. Vere-Jones, An Introduction to the Theory of Point Processes. Vol.I: Elementary Theory and Methods, Springer-Verlag, New York, (2003).

[8] R. del Rio, S. Jitomirskaya, Y. Last and B. Simon, Operators with singular continuous spectrum, $I V$. Hausdorff dimensions, rank one perturbation, and localization, J. Analyse Mathematiques, 69 (1996), 153-200.

[9] I. Guarneri and H. Schulz-Baldes, Lower bounds on wave-packet propagation by packing dimensions of spectral measures, Math. Phys. Elect. J., 5 (1999), 1-16.

[10] E. Habil, Double sequences and double series, IUG Journal of Natural Studies, 14 (2006), 219233.

[11] Y. Last, Quantum dynamics and decomposition of singular continuous spectra, J. Funct. Anal., 142 (1996), 406-445.

[12] P. Mattila, Geometry of Sets and Measures in Euclidean Spaces: Fractals and Rectifiability, Cambridge Univ. Press, Cambridge, (1999).

[13] J.C. Oxtoby, On two theorems of Parthasarathy and Kakutani concerning the shift transformation, Ergodic Theory (Proc. Internat. Sympos., Tulane Univ., New Orleans, La., 1961) Academic Press, New York, (1963), 203-215.

[14] C.A. Rogers and S.J. Taylor, Functions continuous and singular with respect to a Hausdorff measure, Mathematika, 8 (1961), 1-31.

[15] B. Simon, Operators with singular continuous spectrum: I. General operators, Ann. of Math., (2) 141 (1995), 131-145.

[16] L.-S. Young, Dimension, entropy and Lyapunov exponents, Ergod. Th. \& Dynam. Syst., 2 (1982), 109-124. 